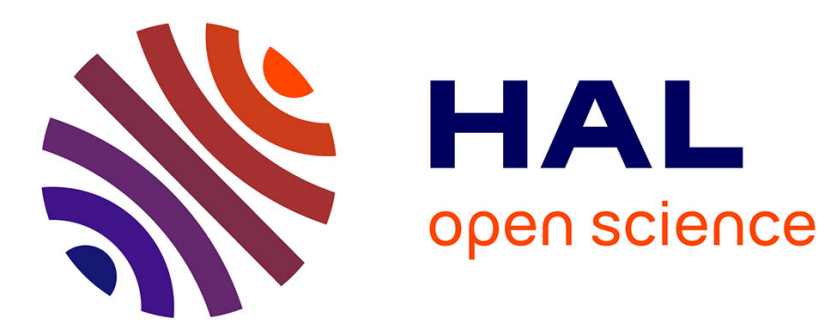

\title{
Static Output Feedback Stabilization of Discrete-Time Linear Systems With Stochastic Dynamics Determined by an Independent Identically Distributed Process
}

Yohei Hosoe, Dimitri Peaucelle

\section{- To cite this version:}

Yohei Hosoe, Dimitri Peaucelle. Static Output Feedback Stabilization of Discrete-Time Linear Systems With Stochastic Dynamics Determined by an Independent Identically Distributed Process. IEEE Control Systems Letters, 2019, 3 (3), pp.673-678. 10.1109/LCSYS.2019.2916289 hal-03026829

\section{HAL Id: hal-03026829 https://hal.science/hal-03026829}

Submitted on 26 Nov 2020

HAL is a multi-disciplinary open access archive for the deposit and dissemination of scientific research documents, whether they are published or not. The documents may come from teaching and research institutions in France or abroad, or from public or private research centers.
L'archive ouverte pluridisciplinaire HAL, est destinée au dépôt et à la diffusion de documents scientifiques de niveau recherche, publiés ou non, émanant des établissements d'enseignement et de recherche français ou étrangers, des laboratoires publics ou privés. 


\title{
Static Output Feedback Stabilization of Discrete-Time Linear Systems with Stochastic Dynamics Determined by an i.i.d. Process
}

\author{
Yohei Hosoe and Dimitri Peaucelle
}

\begin{abstract}
This paper discusses an extension of our earlier results about state feedback stabilization toward static output feedback stabilization for discrete-time linear systems with stochastic dynamics determined by an independent and identically distributed (i.i.d.) process. A completely-linearized necessary and sufficient inequality condition for static output feedback synthesis is known to be difficult to obtain even in the case with deterministic systems. Hence, this paper derives with the so-called $S$-variable approach a bilinear matrix inequality condition that can be effectively solved through exploiting the result of state feedback synthesis. An illustrative numerical example is also given for such synthesis.
\end{abstract}

\section{INTRODUCTION}

Randomness is regarded as one of the important concepts in modern science and technology, with which various kinds of phenomenon have been interpreted and described. Control technology is also no exception, and the demand for approaches that can take into account randomness behind systems is increasing more and more. To make a contribution in this direction of studies, this paper discusses static output feedback stabilization for discrete-time linear systems with dynamics determined by a stochastic process. Such systems are called discrete-time linear random dynamical systems in the field of analytical dynamics [1]. In this paper, we particularly focus on the case that the process determining the system dynamics is independent and identically distributed (i.i.d.) with respect to the discrete time, as in the earlier studies [2], [3], [4].

Markov jump systems [5] are one of the most famous subclasses of random dynamical systems. Although the dynamics of a standard Markov jump system is described with a finite-mode Markov chain, the earlier study [6] succeeded in alleviating this part of assumption so that a more general stationary Markov process can be dealt with in stability analysis. Since i.i.d. processes are a special case of stationary Markov processes, one might consider our arguments would be covered by the results for Markov jump systems. However, the above results on Markov jump systems were actually obtained under an additional assumption that the supports of random coefficient matrices (depending on a Markov process) are bounded, which is needless to use in the i.i.d. process case; instead, a milder assumption is used

This work was partially supported by JSPS KAKENHI Grant Number $17 \mathrm{~K} 14700$.

Y. Hosoe is with the Department of Electrical Engineering, Kyoto University, Nishikyo-ku, Kyoto 615-8510, Japan and with LAAS-CNRS, Univ. Toulouse, CNRS, Toulouse 31400, France hosoeakuee.kyoto-u.ac.jp

D. Peaucelle is with LAAS-CNRS, Univ. Toulouse, CNRS, Toulouse 31400, France peaucelledlaas. fr just for defining second-moment stability (see Assumption 2 introduced later). Hence, the arguments for the above two subclasses do not cover each other, in general. Regarding the relationship of the projection-based control approach [2] with our study for essentially the same systems, the latter can be seen as a redevelopment of the theoretical basis first developed in the former with the recent results on linear matrix inequality (LMI)-based control. A difference can be found, e.g., in the way of dealing with stability; stability is characterized by the spectral radius of some mapping in [2] while it is by a Lyapunov inequality in our study that can be solved as a standard LMI through some pre-calculation of expectation (details will be clearer later).

In [3], [4], we discussed state feedback stabilization as a part of main results. The purpose of this paper is to extend those results toward static output feedback stabilization (under the assumption that the output equation of a system is described with a constant $C$ matrix). It is considered to be unusual in practice that all the system states can be directly measured, and one may have to design an output feedback controller or to use a state observer together with a state feedback controller. This paper is concerned with the former theme (i.e., output feedback synthesis). In the case of deterministic systems, complete linearization of the inequality condition for static output feedback stabilization controller synthesis is known to be difficult [7]. Hence, a similar difficulty finally remains also in the present arguments for stochastic systems. However, at least the same level of results as the case of deterministic systems is obtained even in such a situation through using the $S$-variable approach [8], which are expected to be sufficiently practical as is the case with deterministic systems.

The results in this paper could be used, e.g., for taking into account the effect of randomness of packet interarrival times in control for networked systems; an associated numerical example about random aperiodic sampling (i.e., a random version of aperiodic sampling [9]) was dealt with in [4]. Another prospective application would be the combination of our theory with statistical approaches for parameter estimation such as the ensemble Kalman filter [10], [11]. As is well known, the estimated parameters in modeling more or less fluctuate in practice. Our framework has a potential of dealing with such fluctuations more effectively in control than deterministic approaches.

This paper is organized as follows. Section II describes the system to be dealt with in this paper, and introduces some assumptions and the definition of exponential secondmoment stability for the system. Section III briefly reviews 
a Lyapunov inequality characterizing the stability as well as an inequality condition for synthesis of stabilizing state feedback. Then, Section IV discusses main results about static output feedback synthesis. Since the technique of change of variables used in the earlier study on state feedback synthesis is difficult to employ in the case of output feedback directly, we consider introducing an auxiliary variable called an $S$ variable for deriving a new inequality condition. Although we will not obtain a completely-linearized inequality condition even with the $S$-variable, the new condition can be effectively solved by exploiting the result of state feedback synthesis appropriately. We also describe the details of this, and propose a two-step synthesis method using our inequality conditions. An illustrative numerical example of our synthesis is given in Section V.

We use the following notation in this paper. The set of real numbers, that of positive real numbers and that of nonnegative integers are denoted by $\mathbf{R}, \mathbf{R}_{+}$and $\mathbf{N}_{0}$, respectively. The set of $n$-dimensional real column vectors and that of $m \times n$ real matrices are denoted by $\mathbf{R}^{n}$ and $\mathbf{R}^{m \times n}$, respectively. The set of $n \times n$ positive definite matrices is denoted by $\mathbf{S}_{+}^{n \times n}$. The identity matrix of size $n$ is denoted by $I_{n}$. The Euclidean norm of the vector $(\cdot)$ is denoted by $\|(\cdot)\|$. The vectorization of the matrix $(\cdot)$ in the row direction is denoted by $\operatorname{row}(\cdot)$, i.e., $\operatorname{row}(\cdot):=\left[\operatorname{row}_{1}(\cdot), \ldots, \operatorname{row}_{m}(\cdot)\right]$, where $m$ is the number of rows of the matrix and $\operatorname{row}_{i}(\cdot)$ denotes the $i$ th row. The Kronecker product is denoted by $\otimes$. The (block) diagonal matrix is denoted by $\operatorname{diag}(\cdot)$. For the real square matrix $M, \operatorname{He}(M):=M+M^{T}$, where $M^{T}$ denotes the transpose of $M$. The expectation (i.e., the expected value) of the random variable $(\cdot)$ is denoted by $E[(\cdot)]$; this notation is also used for the expectation of a random matrix. If $s$ is a random variable obeying the distribution $\mathcal{D}$, then we represent it as $s \sim \mathcal{D}$.

\section{Discrete-Time Linear Stochastic Systems And SECOND-MOMENT STABility}

\section{A. Discrete-Time Linear Systems with Stochastic Dynamics Determined by an i.i.d. Process}

Let us consider the $Z$-dimensional discrete-time stochastic process $\xi=\left(\xi_{k}\right)_{k \in \mathbf{N}_{0}}$ satisfying the following assumption.

Assumption 1: $\xi_{k}$ is independent and identically distributed (i.i.d.) with respect to the discrete time $k \in \mathbf{N}_{0}$.

This assumption naturally makes $\xi$ stationary and ergodic [12]. The support of $\xi_{k}$ is denoted by $\boldsymbol{\Xi}$. By definition, $\boldsymbol{\Xi} \subset$ $\mathbf{R}^{Z}$, and $\boldsymbol{\Xi}$ corresponds to the set of values that $\xi_{k}$ can take (at each $k$ ).

With such a process $\xi$, consider the discrete-time linear random dynamical system

$$
\begin{aligned}
& x_{k+1}=A\left(\xi_{k}\right) x_{k}+B\left(\xi_{k}\right) u_{k}, \\
& y_{k}=C x_{k}
\end{aligned}
$$

where $x_{k} \in \mathbf{R}^{n}, u_{k} \in \mathbf{R}^{m_{u}}, y_{k} \in \mathbf{R}^{m_{y}}, A: \boldsymbol{\Xi} \rightarrow \mathbf{R}^{n \times n}$, $B: \boldsymbol{\Xi} \rightarrow \mathbf{R}^{n \times m_{u}}, C \in \mathbf{R}^{m_{y} \times n}$, and the initial state $x_{0}$ is assumed to be deterministic. The dynamics of this system is determined by the random coefficient matrix $A\left(\xi_{k}\right)$, which is i.i.d. with respect to $k$ because of Assumption 1. Hence, we call the system having such dynamics a discrete-time linear system with stochastic dynamics determined by an i.i.d. process. The representation of this $A$ matrix is indeed general in the sense that any random matrix (denoted by $A_{k}$ ) can be represented in that form with appropriate $\xi$; we can always take $A(\cdot)$ and $\xi$ such that $A\left(\xi_{k}\right)=A_{k}$ (under the i.i.d. assumption on $A_{k}$ and $\xi_{k}$ ). A similar comment also applies to $B\left(\xi_{k}\right)$. Although $C$ in (2) is confined to be a deterministic constant matrix, this is natural, e.g., when a deterministic filter is connected to the output of the system.

\section{B. Closed-Loop System and Second-Moment Stability}

As a controller for the system described in the preceding subsection, let us consider the output feedback

$$
u_{k}=K y_{k}
$$

with static time-invariant gain $K \in \mathbf{R}^{m_{u} \times m_{y}}$. Then, the closed-loop system becomes

$$
x_{k+1}=A_{\text {of }}\left(\xi_{k}\right) x_{k}, \quad A_{\text {of }}\left(\xi_{k}\right)=A\left(\xi_{k}\right)+B\left(\xi_{k}\right) K C .
$$

To define second-moment stability for this system, we introduce the following assumption (which is actually a minimal requirement for the stability definition, although the details are omitted).

Assumption 2: The squares of elements of $A\left(\xi_{k}\right)$ and $B\left(\xi_{k}\right)$ are all Lebesgue integrable, i.e.,

$$
E\left[A_{i j}\left(\xi_{k}\right)^{2}\right]<\infty, \quad E\left[B_{i j}\left(\xi_{k}\right)^{2}\right]<\infty,
$$

where $A_{i j}\left(\xi_{k}\right)$ and $B_{i j}\left(\xi_{k}\right)$ represent the $(i, j)$-entries of $A\left(\xi_{k}\right)$ and $B\left(\xi_{k}\right)$, respectively.

For each fixed $K$, this assumption ensures the squares of elements of $A_{\mathrm{of}}\left(\xi_{k}\right)$ are also Lebesgue integrable. Under Assumptions 1 and 2, we define exponential second-moment stability (i.e., exponential mean square stability) [13] as follows.

Definition 1: The system (4) with a fixed $K$ satisfying Assumptions 1 and 2 is said to be exponentially stable in the second moment if there exist $a \in \mathbf{R}_{+}$and $\lambda \in(0,1)$ such that

$$
\sqrt{E\left[\left\|x_{k}\right\|^{2}\right]} \leq a\left\|x_{0}\right\| \lambda^{k} \quad\left(\forall k \in \mathbf{N}_{0}, \forall x_{0} \in \mathbf{R}^{n}\right) .
$$

In this paper, we discuss a method of designing $K$ that stabilizes the closed-loop system (4) in the sense of Definition 1 under Assumptions 1 and 2.

\section{EARlier RESUlts on Stabilizing State FEEDBACK SYNTHESIS}

If all the system states can be measured at each time step (i.e., $m_{y}=n$ and $C=I_{n}$ in (2)), the present output feedback stabilization problem reduces to that of designing a stabilizing state feedback gain. Such a special case was already studied in [4], and this section reviews the earlier results before proceeding to the main discussions on static output feedback stabilization; those results themselves will also play an important role in the output feedback stabilization discussed later. 
Let us consider the state feedback

$$
u_{k}=F x_{k}
$$

with static time-invariant gain $F \in \mathbf{R}^{m_{u} \times n}$, and the associated closed-loop system

$$
x_{k+1}=A_{\mathrm{sf}}\left(\xi_{k}\right) x_{k}, \quad A_{\mathrm{sf}}\left(\xi_{k}\right)=A\left(\xi_{k}\right)+B\left(\xi_{k}\right) F .
$$

We see that the output feedback stated in the preceding section reduces to the above state feedback under $K=F$ if $m_{y}=n$ and $C=I_{n}$. This implies that Assumption 2 also ensures the squares of elements of $A_{\mathrm{sf}}\left(\xi_{k}\right)$ are Lebesgue integrable for each $F$, and hence, the second-moment stability can be defined for system (8) in a fashion similar to Definition 1. Then, the following theorem is known to hold (see Theorems 2 and 3 in [4]), which gives a Lyapunov inequality condition for second-moment stability.

Theorem 1: Suppose the open-loop system (1) satisfies Assumptions 1 and 2. For given $F \in \mathbf{R}^{m_{u} \times n}$, the following two conditions are equivalent.

1) The closed-loop system (8) is exponentially stable in the second moment.

2) There exist $P \in \mathbf{S}_{+}^{n \times n}$ and $\lambda \in(0,1)$ such that

$$
E\left[\lambda^{2} P-A_{\mathrm{sf}}\left(\xi_{0}\right)^{T} P A_{\mathrm{sf}}\left(\xi_{0}\right)\right]>0 .
$$

With the Lyapunov inequality (9), we can further obtain the following theorem [4], which gives an inequality condition for synthesis of stabilizing state feedback.

Theorem 2: Suppose the open-loop system (1) satisfies Assumptions 1 and 2. There exists a gain $F$ such that the closed-loop system (8) is exponentially stable in the second moment if and only if there exist $X \in \mathbf{S}_{+}^{n \times n}, Y \in \mathbf{R}^{m_{u} \times n}$ and $\lambda \in(0,1)$ satisfying

$$
\left[\begin{array}{cc}
\lambda^{2} X & * \\
\bar{G}_{A}^{\prime} X+\bar{G}_{B}^{\prime} Y & X \otimes I_{\bar{n}}
\end{array}\right]>0
$$

for $\bar{G}_{A}^{\prime}$ and $\bar{G}_{B}^{\prime}$ given by

$$
\begin{aligned}
& \bar{G}_{A}^{\prime}:=\left[\bar{G}_{A 1}^{T}, \ldots, \bar{G}_{A n}^{T}\right]^{T} \in \mathbf{R}^{n \bar{n} \times n}, \\
& \bar{G}_{B}^{\prime}:=\left[\bar{G}_{B 1}^{T}, \ldots, \bar{G}_{B n}^{T}\right]^{T} \in \mathbf{R}^{n \bar{n} \times m_{u}}, \\
& \bar{G}^{\prime}=:\left[\bar{G}_{A 1}, \ldots, \bar{G}_{A n}, \bar{G}_{B 1}, \ldots, \bar{G}_{B n}\right] \\
& \left(\bar{G}_{A i} \in \mathbf{R}^{\bar{n} \times n}, \bar{G}_{B i} \in \mathbf{R}^{\bar{n} \times m_{u}}(i=1, \ldots, n)\right)
\end{aligned}
$$

with a matrix $\bar{G} \in \mathbf{R}^{\bar{n} \times\left(n+m_{u}\right) n}\left(\bar{n} \leq\left(n+m_{u}\right) n\right)$ satisfying

$$
\begin{aligned}
& \bar{G}^{T} \bar{G}=E\left[\left[\operatorname{row}\left(A\left(\xi_{0}\right)\right), \operatorname{row}\left(B\left(\xi_{0}\right)\right)\right]^{T}\right. \\
& \left.\cdot\left[\operatorname{row}\left(A\left(\xi_{0}\right)\right), \operatorname{row}\left(B\left(\xi_{0}\right)\right)\right]\right] .
\end{aligned}
$$

In particular, $F=Y X^{-1}$ is one such stabilizing gain.

While the Lyapunov inequality (9) involved decision variables contained in the expectation operation, the decision variables in (10) are all uncontained in the expectation operation. Hence, once $\bar{G}_{A}^{\prime}$ and $\bar{G}_{B}^{\prime}$ are calculated, (10) can be solved as a standard LMI for each fixed $\lambda$. By minimizing $\lambda$ through a bisection method with respect to $\lambda^{2}$ under such an LMI, we can obtain a stabilizing gain that is optimal in the sense of exponential second-moment stability; the minimal $\lambda$ corresponds to that in (6), which further corresponds to the convergence rate of $\sqrt{E\left[\left\|x_{k}\right\|^{2}\right]}$ with respect to $k$.

Remark 1: In [4], the Lyapunov inequality was shown with the non-strict inequality sign, unlike (9). However, this difference does not make any problem since for fixed $P$, there exists $\lambda \in(0,1)$ satisfying (9) if and only if there exists $\lambda \in(0,1)$ satisfying its non-strict version; this can be confirmed with $P>0$ and the gap between $\lambda$ and 1 . Since we need to deal with strict inequalities in the output feedback synthesis later, we introduced the strict version (9) of Lyapunov inequality in this section.

\section{Static Output Feedback Stabilization}

As reviewed in the preceding section, we can easily design a stabilizing state feedback gain $F$ by using Theorem 2 . One of the implicitly used tricks in deriving Theorem 2 was the change of variables $Y=F X$ for $X=P^{-1}$ in (10), which has been used also in the case of deterministic systems. Such conventional techniques and some other key ideas associated with (11)-(14) led us to Theorem 2 in the earlier study. However, that change of variables is difficult to use in the case of the present static output feedback stabilization. This section first briefly describes this issue, and then shows an inequality condition for static output feedback stabilization that is derived with a different approach.

\section{A. Difficulty in Change of Variables}

Since the squares of elements of $A_{\mathrm{of}}\left(\xi_{k}\right)$ are Lebesgue integrable under Assumptions 1 and 2, the stability of system (4) can be characterized by the following Lyapunov inequality as in Theorem 1.

$$
E\left[\lambda^{2} P-A_{\text {of }}\left(\xi_{0}\right)^{T} P A_{\text {of }}\left(\xi_{0}\right)\right]>0 .
$$

Then, it can be shown from the arguments in [4] that

$$
\begin{aligned}
& E\left[A\left(\xi_{0}\right)^{T} P A\left(\xi_{0}\right)\right]=\bar{G}_{A}^{\prime T}\left(P \otimes I_{\bar{n}}\right) \bar{G}_{A}^{\prime}, \\
& E\left[A\left(\xi_{0}\right)^{T} P B\left(\xi_{0}\right)\right]=\bar{G}_{A}^{\prime T}\left(P \otimes I_{\bar{n}}\right) \bar{G}_{B}^{\prime}, \\
& E\left[B\left(\xi_{0}\right)^{T} P B\left(\xi_{0}\right)\right]=\bar{G}_{B}^{\prime T}\left(P \otimes I_{\bar{n}}\right) \bar{G}_{B}^{\prime}
\end{aligned}
$$

(with which Theorem 2 was derived); although $\bar{n}$ was fixed at $\left(n+m_{u}\right) n$ in [4], it may actually be arbitrary as in Theorem 2 of the present paper as long as (14) is satisfied. By using (16)-(18), the Lyapunov inequality (15) can be equivalently rewritten as

$$
\lambda^{2} P-\left(\bar{G}_{A}^{\prime}+\bar{G}_{B}^{\prime} K C\right)^{T}\left(P \otimes I_{\bar{n}}\right)\left(\bar{G}_{A}^{\prime}+\bar{G}_{B}^{\prime} K C\right)>0 .
$$

This leads us to the following lemma.

Lemma 1: Suppose the open-loop system (1) and (2) satisfies Assumptions 1 and 2. For given $K \in \mathbf{R}^{m_{u} \times m_{y}}$, the closed-loop system (4) is exponentially stable in the second moment if and only if there exist $P \in \mathbf{S}_{+}^{n \times n}$ and $\lambda \in(0,1)$ satisfying (19) for $\bar{G}_{A}^{\prime}$ and $\bar{G}_{B}^{\prime}$ given by (11)-(14).

Hence, if (19) can be linearized as in Theorem 2, we can easily design a static output feedback. However, the Schur 
complement technique [14] and the congruence transformation using $\operatorname{diag}\left(X, X \otimes I_{\bar{n}}\right)$ for $X=P^{-1}$ only leads us to the following inequality from (19).

$$
\left[\begin{array}{cc}
\lambda^{2} X & * \\
\bar{G}_{A}^{\prime} X+\bar{G}_{B}^{\prime} K C X & X \otimes I_{\bar{n}}
\end{array}\right]>0
$$

Since $C$ is generally not a nonsingular square matrix in the present output feedback control problem, we cannot apply the change of variables to the product $K C X$ in the above inequality. This is the issue in the output feedback synthesis, which does not occur in the state feedback synthesis (see, e.g., [7] for the associated discussions on deterministic systems). Even if we try to solve the bilinear matrix inequality (BMI) (20) iteratively by fixing a part of decision variables (i.e., $K$ or $X$ ) at each iteration, the way of selecting the initial value for the variables becomes a problem; we have no reasonable way of the selection for $K$ and $X$ in (20). Since synthesis results obtained with BMIs do generally depend on initial values, the above issue is significant. To alleviate this issue, the next subsection shows an inequality condition with what we call an $S$-variable [8].

\section{B. Inequality Condition Derived with S-variable Approach and Two-Step Synthesis}

We first show the following lemma, which gives another inequality condition for stability of closed-loop system (4).

Lemma 2: Suppose the open-loop system (1) and (2) satisfies Assumptions 1 and 2. For given $K \in \mathbf{R}^{m_{u} \times m_{y}}$, $P \in \mathbf{S}_{+}^{n \times n}$ and $\lambda \in(0,1)$, the following two conditions are equivalent.

1) The inequality (19) is satisfied.

2) There exists $S \in \mathbf{R}^{\left(n+m_{u}\right) \times m_{u}}$ satisfying

$$
\begin{aligned}
& {\left[\begin{array}{cc}
\bar{G}_{A}^{\prime} & \bar{G}_{B}^{\prime} \\
I_{n} & 0
\end{array}\right]^{T}\left[\begin{array}{cc}
P \otimes I_{\bar{n}} & 0 \\
0 & -\lambda^{2} P
\end{array}\right]\left[\begin{array}{cc}
\bar{G}_{A}^{\prime} & \bar{G}_{B}^{\prime} \\
I_{n} & 0
\end{array}\right]} \\
& <\operatorname{He}\left(S\left[\begin{array}{ll}
K C & -I_{m_{u}}
\end{array}\right]\right) \text {. }
\end{aligned}
$$

Proof: $2 \Rightarrow 1$ : Let us denote the left-hand side of (21) by $Q$. Then, by pre- and post-multiplying $\left[I_{n}, C^{T} K^{T}\right]$ and its transpose on (21), we have

$$
\left[\begin{array}{c}
I_{n} \\
K C
\end{array}\right]^{T} Q\left[\begin{array}{c}
I_{n} \\
K C
\end{array}\right]<0
$$

which is equivalent to (19) (regardless of $S$ ).

$1 \Rightarrow 2$ : Since (19) can be rewritten as (22), it follows from Finsler's lemma [8] that (19) holds if and only if there exists $\tau \in \mathbf{R}_{+}$such that

$$
Q<\tau\left[\begin{array}{c}
C^{T} K^{T} \\
-I_{m_{u}}
\end{array}\right]\left[\begin{array}{ll}
K C & -I_{m_{u}}
\end{array}\right] .
$$

This is nothing but (21) with

$$
S=\frac{\tau}{2}\left[\begin{array}{c}
C^{T} K^{T} \\
-I_{m_{u}}
\end{array}\right] .
$$

This completes the proof.

The auxiliary variable $S$ in (21) is called an $S$-variable [8]. By using $S$-variables, various kinds of inequality condition have been derived for control of deterministic systems; roughly speaking, the $S$-variable approach is an approach to introducing auxiliary variables in matrix inequalities based on Finsler's lemma (or the elimination lemma). Lemma 2 implies that this approach is useful even for the present stochastic systems control (in which the expectation operation has to be dealt with appropriately). For $F \in \mathbf{R}^{m_{u} \times n}$ and $S_{2} \in \mathbf{R}^{m_{u} \times m_{u}}$, let us represent the $S$-variable in (21) by

$$
S=\left[\begin{array}{c}
F^{T} S_{2} \\
-S_{2}
\end{array}\right]
$$

without loss of generality; $S_{2}$ is nonsingular when (21) is satisfied, which can be confirmed from the lower-right block and $P>0$ (to ensure this nonsingularity, the inequality sign in the Lyapunov inequality needed to be strict). Then, (21) can be rewritten as

$$
\begin{aligned}
& {\left[\begin{array}{cc}
\bar{G}_{A}^{\prime} & \bar{G}_{B}^{\prime} \\
I_{n} & 0
\end{array}\right]^{T}\left[\begin{array}{cc}
P \otimes I_{\bar{n}} & 0 \\
0 & -\lambda^{2} P
\end{array}\right]\left[\begin{array}{cc}
\bar{G}_{A}^{\prime} & \bar{G}_{B}^{\prime} \\
I_{n} & 0
\end{array}\right] } \\
< & \operatorname{He}\left(\left[\begin{array}{c}
F^{T} \\
-I_{m_{u}}
\end{array}\right]\left[\begin{array}{ll}
S_{2} K C & -S_{2}
\end{array}\right]\right) .
\end{aligned}
$$

Hence, this, together with Lemmas 1 and 2 and the change of variables $S_{1}=S_{2} K$, leads us to the following theorem.

Theorem 3: Suppose the open-loop system (1) and (2) satisfies Assumptions 1 and 2. There exists a gain $K$ such that the closed-loop system (4) is exponentially stable in the second moment if and only if there exist $F \in \mathbf{R}^{m_{u} \times n}$, $S_{1} \in \mathbf{R}^{m_{u} \times m_{y}}, S_{2} \in \mathbf{R}^{m_{u} \times m_{u}}, P \in \mathbf{S}_{+}^{n \times n}$ and $\lambda \in(0,1)$ satisfying

$$
\begin{aligned}
& \quad\left[\begin{array}{cc}
\bar{G}_{A}^{\prime} & \bar{G}_{B}^{\prime} \\
I_{n} & 0
\end{array}\right]^{T}\left[\begin{array}{cc}
P \otimes I_{\bar{n}} & 0 \\
0 & -\lambda^{2} P
\end{array}\right]\left[\begin{array}{cc}
\bar{G}_{A}^{\prime} & \bar{G}_{B}^{\prime} \\
I_{n} & 0
\end{array}\right] \\
< & \operatorname{He}\left(\left[\begin{array}{c}
F^{T} \\
-I_{m_{u}}
\end{array}\right]\left[\begin{array}{ll}
S_{1} C & -S_{2}
\end{array}\right]\right)
\end{aligned}
$$

for $\bar{G}_{A}^{\prime}$ and $\bar{G}_{B}^{\prime}$ given by (11)-(14). In particular, $S_{2}$ is nonsingular, and $K=S_{2}^{-1} S_{1}$ is one such stabilizing gain.

As we can see, the inequality condition (27) is still bilinear. However, if we fix $F$, the inequality becomes an LMI. The important point here is that the variable $F$ is not a Lyapunov matrix but an auxiliary variable, and hence, the influence of fixing it is relatively limited. In addition, a reasonable way of selecting $F$ actually exists, which is related to the state feedback synthesis as indicated by the used symbol for the variable. To see this, we show the following lemma.

Lemma 3: Suppose the open-loop system (1) and (2) satisfies Assumptions 1 and 2. For given $F \in \mathbf{R}^{m_{u} \times n}$, $P \in \mathbf{S}_{+}^{n \times n}$ and $\lambda \in(0,1)$, there exist $S_{1} \in \mathbf{R}^{m_{u} \times m_{y}}$ and $S_{2} \in \mathbf{R}^{m_{u} \times m_{u}}$ satisfying (27) only if (9) holds.

Proof: By pre- and post-multiplying $\left[I_{n}, F^{T}\right]$ and its transpose on (27), we have (9) under (16)-(18). This implies that (27) can have a solution only if (9) holds.

From this lemma, it is meaningless in (27) to consider $F$ that does not satisfy (9), which in turn implies that selecting a stabilizing state feedback gain as the variable $F$ is reasonable. Although such selection of $F$ does not necessarily 
lead to a globally optimal (or near) solution, a similar idea is empirically known to be effective in the case of deterministic systems [7]. Hence, this paper also exploits this idea and proposes the following two-step synthesis method for static output feedback stabilization controller synthesis.

Step 1: Minimize $\lambda$ such that there exist $X \in \mathbf{S}_{+}^{n \times n}$ and $Y \in \mathbf{R}^{m_{u} \times n}$ satisfying (10) through a bisection method with respect to $\lambda^{2}$. If $\lambda<1$, take $F=Y X^{-1}$, and proceed to the next step; if not, the problem is unsolvable by Lemma 3.

Step 2: For fixed $F$, minimize $\lambda$ such that there exist $S_{1} \in$ $\mathbf{R}^{m_{u} \times m_{y}}, S_{2} \in \mathbf{R}^{m_{u} \times m_{u}}$ and $P \in \mathbf{S}_{+}^{n \times n}$ satisfying (27) through a bisection method with respect to $\lambda^{2}$. If $\lambda<1$, then $K=S_{2}^{-1} S_{1}$ is a stabilizing output feedback gain.

Although we did not refer to the iterations about the products of $F, S_{1}$ and $S_{2}$ in (27) in the above method, we can try them if the minimal $\lambda$ does not become sufficiently small with the fixed $F$.

\section{About Assumption on Constant C Matrix}

Before closing this section, we would like to make remarks on the inequality condition (27), which facilitates understanding of the difference between the present study on stochastic systems and those for deterministic systems. With (16)-(18), we can rewrite (27) as

$$
\begin{aligned}
& E\left[\left[\begin{array}{cc}
A\left(\xi_{0}\right) & B\left(\xi_{0}\right) \\
I_{n} & 0
\end{array}\right]^{T}\left[\begin{array}{cc}
P & 0 \\
0 & -\lambda^{2} P
\end{array}\right]\left[\begin{array}{cc}
A\left(\xi_{0}\right) & B\left(\xi_{0}\right) \\
I_{n} & 0
\end{array}\right]\right] \\
& <E\left[\operatorname{He}\left(\left[\begin{array}{c}
F^{T} \\
-I_{m_{u}}
\end{array}\right]\left[\begin{array}{ll}
S_{1} C & -S_{2}
\end{array}\right]\right)\right] \text {. }
\end{aligned}
$$

This is actually a straightforward representation of the inequality condition that can be first anticipated from the form of the expectation-based Lyapunov inequality (15) with knowledge of LMIs for deterministic systems. By pre- and post-multiplying $\left[I_{n}, C^{T} K^{T}\right]$ and its transpose on (28) (as in the proof of Lemma 2), we obtain

$$
\begin{array}{r}
E\left[\left[\begin{array}{c}
A_{\mathrm{of}}\left(\xi_{0}\right) \\
I_{n}
\end{array}\right]^{T}\left[\begin{array}{cc}
P & 0 \\
0 & -\lambda^{2} P
\end{array}\right]\left[\begin{array}{c}
A_{\mathrm{of}}\left(\xi_{0}\right) \\
I_{n}
\end{array}\right]\right] \\
<E\left[\operatorname{He}\left(\left(F^{T}-C^{T} K^{T}\right)\left(S_{1} C-S_{2} K C\right)\right)\right]
\end{array}
$$

with (4). Since the right-hand side of this inequality becomes 0 for $K=S_{2}^{-1} S_{1}$, the inequality reduces to (15). Although this conclusion itself was already led in Theorem 3, the above arguments provide us with more insight into the influence of the presence of $\xi$ in our system. To see this, let us temporarily consider the case where the $C$ matrix in (2) is given by $C\left(\xi_{k}\right)$ (i.e., a random matrix depending on $\xi_{k}$ ). Then, one might consider (28) with $C$ replaced by $C\left(\xi_{0}\right)$ could be the corresponding inequality condition for output feedback synthesis. Unfortunately, however, the arguments for stochastic systems are not so simple, and we soon notice that the dependence of $A\left(\xi_{0}\right), B\left(\xi_{0}\right)$ and $C\left(\xi_{0}\right)$ becomes a problem in obtaining (29) (i.e., (15)) with $C$ replaced by $C\left(\xi_{0}\right)$; for random variables $s_{1}$ and $s_{2}, E\left[s_{1}\right] E\left[s_{2}\right]$ is not equal to $E\left[s_{1} s_{2}\right]$, in general. This is actually the reason why we had to confine ourselves to the case where the $C$ matrix is given by a constant matrix in this paper.
In the case of deterministic systems, it is known that stability of the system $x_{k+1}=A_{\mathrm{d}} x_{k}$ with the $A$ matrix $A_{\mathrm{d}}$ is equivalent to that of the system $\eta_{k+1}=A_{\mathrm{d}}^{T} \eta_{k}$ (i.e., the transposed system). Hence, a similar idea could be used also in the case of our stochastic systems; in that case, we might be able to deal with the random $C$ matrix $C\left(\xi_{0}\right)$ through alternatively confining the $B$ matrix to a constant matrix. However, the arguments for this direction is also not simple, and the expectation operation in inequality conditions causes an issue when we transform them with conventional LMI techniques; this issue is related to the non-square $\bar{G}_{A}^{\prime}$ and the extended Lyapunov matrix $P \otimes I_{\bar{n}}$ in (27). Resolving this issue is left for future work.

\section{Numerical Example}

This section illustrates our synthesis approach through a numerical example. Let us consider the two-dimensional random process $\xi_{k}=\left[\xi_{1 k}, \xi_{2 k}\right]^{T}$ satisfying Assumptions 1 and 2 whose distribution is given by

$$
\xi_{1 k} \sim \mathcal{N}\left(0,0.2^{2}\right), \quad \xi_{2 k} \sim \mathcal{U}(-0.5,0.5),
$$

where $\mathcal{N}\left(0,0.2^{2}\right)$ and $\mathcal{U}(-0.5,0.5)$ denote the normal distribution with mean 0 and standard deviation 0.2 and the continuous uniform distribution with minimum -0.5 and maximum 0.5 , respectively. We assume that $\xi_{1 k}$ and $\xi_{2 k}$ are also independent of each other at each $k$. Then, let us further consider the system (1) and (2) with coefficients

$$
\begin{aligned}
& A\left(\xi_{k}\right)=\left[\begin{array}{ccc}
1.3+\xi_{2 k} & 0.8+\xi_{1 k} & -0.5 \\
0.5 & 0.3+\xi_{1 k} \xi_{2 k} & -1.2+\xi_{1 k}^{2} \\
-0.2 & 0.8 & 0.6
\end{array}\right], \\
& B\left(\xi_{k}\right)=\left[\begin{array}{lll}
0 & \xi_{1 k} & 1
\end{array}\right]^{T}, \quad C=\left[\begin{array}{ccc}
1 & 0 & 0 \\
0 & 0 & 1
\end{array}\right] .
\end{aligned}
$$

This system can be confirmed to be unstable by Theorem 1 with $F=0$.

For the above system, we first design a state feedback gain based on Theorem 2, which corresponds to Step 1 of our synthesis method. The expectation in (14) can be calculated with MATLAB and Symbolic Math Toolbox. Since $\bar{n}$ is arbitrary, taking it as the rank of the expectation (i.e., constant matrix) is reasonable. The corresponding $\bar{G}$ (and hence, $\bar{G}_{A}^{\prime}$ and $\bar{G}_{B}^{\prime}$ ) can be constructed with the singular value decomposition. In addition, LMIs can be solved with MATLAB, YALMIP [15] and SDPT3 [16]. In our calculation, $\bar{n}$ in (11) and (12) was 5. Through minimizing $\lambda$ with respect to (10), we obtained $\lambda=0.8174$, and $X$ and $Y$ leading to

$$
F=Y X^{-1}=\left[\begin{array}{lll}
2.3120 & 0.4735 & -2.2271
\end{array}\right] \text {. }
$$

Since the above $\lambda$ is less than 1 , the closed-loop system (8) with this gain is stable by Theorem 2 . Since the condition in the theorem is necessary and sufficient, this value of $\lambda$ is optimal in the sense of state feedback stabilization using a static gain. This further ensures that the above value is a lower bound of $\lambda$ that can be achieved by static output feedback (recall Lemma 3), which we next design.

The matrices $\bar{G}_{A}^{\prime}$ and $\bar{G}_{B}^{\prime}$ were already calculated in the above state feedback synthesis, and we can use them also for 

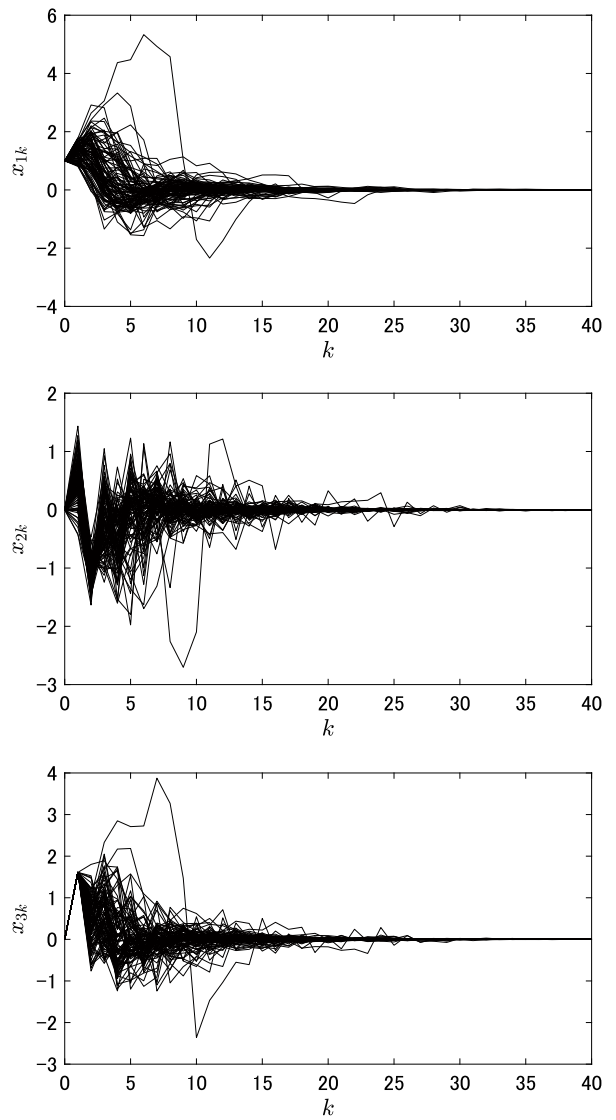

Fig. 1. Overlays of initial value response of output feedback system generated with 100 sample paths of $\xi$ and $x_{0}=[1,0,0]^{T}$.

the output feedback synthesis based on Theorem 3, which corresponds to Step 2 of our synthesis method. We fixed $F$ obtained above, and minimized $\lambda$ with respect to (27). Then, we obtained $\lambda=0.8614$, and $S_{1}$ and $S_{2}$ leading to

$$
K=S_{2}^{-1} S_{1}=\left[\begin{array}{ll}
1.8093 & -1.8487
\end{array}\right] \text {. }
$$

Since the above $\lambda$ is less than 1, the closed-loop system (4) with this gain is stable by Theorem 3. Hence, our two-step synthesis succeeded in stabilization for the present example. The initial value response of state of the closed-loop system with $x_{0}=[1,0,0]^{T}$ became as in Fig. 1; this figure shows the overlays of the response obtained with 100 sample paths of $\xi$.

Since the result $\lambda=0.8614$ of output feedback synthesis is relatively close to the result $\lambda=0.8174$ of state feedback synthesis, the room for further improvement about $\lambda$ (i.e., the convergence rate of $\left.\sqrt{E\left[\left\|x_{k}\right\|^{2}\right]}\right)$ in the output feedback synthesis is considered to be not large in the present example. For reference, with an additional iteration for solving (27), we obtained $\lambda=0.8587$ with a slightly different gain.

\section{CONCLUSIONS}

In this paper, we derived an inequality condition for static output feedback stabilization of discrete-time linear systems with stochastic dynamics determined by an i.i.d. process. Since the complete linearization of the inequality condition was difficult as is the case with deterministic systems, we propose a two-step synthesis method in which a state feedback gain is used as the fixed parameter in the inequality condition. A lemma was also provided for showing reasonability of such synthesis.

As a future issue, the case of the random $C$ matrix is remaining. In addition, the extension of the results in this paper toward robust output stabilization might be possible through exploiting the idea of random polytopes [17] as in the state feedback synthesis in [3]; random polytopes can be used for representing uncertainties, e.g., in mean and variance of random matrices.

\section{REFERENCES}

[1] L. Arnold, Random Dynamical Systems. Berlin Heidelberg, Germany: Springer-Verlag, 1998

[2] W. L. De Koning, "Compensatability and optimal compensation of systems with white parameters," IEEE Transactions on Automatic Control, vol. 37, no. 5, pp. 579-588, 1992.

[3] Y. Hosoe, T. Hagiwara, and D. Peaucelle, "Robust stability analysis and state feedback synthesis for discrete-time systems characterized by random polytopes," IEEE Transactions on Automatic Control, vol. 63 , no. 2, pp. 556-562, 2018.

[4] Y. Hosoe and T. Hagiwara, "Equivalent stability notions, Lyapunov inequality, and its application in discrete-time linear systems with stochastic dynamics determined by an i.i.d. process," IEEE Transactions on Automatic Control, to appear.

[5] O. L. V. Costa, M. D. Fragoso, and R. P. Marques, Discrete-Time Markov Jump Linear Systems. London, UK: Springer-Verlag, 2005.

[6] O. L. V. Costa and D. Z. Figueiredo, "Stochastic stability of jump discrete-time linear systems with Markov chain in a general Borel space," IEEE Transactions on Automatic Control, vol. 59, no. 1, pp. 223-227, 2014.

[7] D. Arzelier, F. Dabbene, S. Formentin, D. Peaucelle, and L. Zaccarian, "Robust static output feedback design with deterministic and probabilistic certificates," in Uncertainty in Complex Networked Systems: In Honor of Roberto Tempo, T. Başar, Ed. Cham, Switzerland: Springer 2018, pp. 121-148.

[8] Y. Ebihara, D. Peaucelle, and D. Arzelier, S-Variable Approach to LMI-Based Robust Control. London, UK: Springer-Verlag, 2015.

[9] L. Hetel, C. Fiter, H. Omran, A. Seuret, E. Fridman, J.-P. Richard, and S. I. Niculescu, "Recent developments on the stability of systems with aperiodic sampling: An overview," Automatica, vol. 76, no. 2, pp. 309-335, 2017.

[10] G. Evensen, "The ensemble Kalman filter: Theoretical formulation and practical implementation," Ocean Dynamics, vol. 53, no. 4, pp. 343-367, 2003.

[11] G. Evensen, Data Assimilation: The Ensemble Kalman Filter, 2nd ed. Berlin Heidelberg, Germany: Springer-Verlag, 2009.

[12] A. Klenke, Probability Theory: A Comprehensive Course, 2nd ed. London, UK: Springer-Verlag, 2014.

[13] F. Kozin, "A survey of stability of stochastic systems," Automatica, vol. 5, no. 1, pp. 95-112, 1969.

[14] S. Boyd, L. El Ghaoui, E. Feron, and V. Balakrishnan, Linear Matrix Inequalities in System and Control Theory. Philadelphia, PA, USA: SIAM, 1994

[15] J. Löfberg, "YALMIP: A toolbox for modeling and optimization in MATLAB," in Proc. 2004 IEEE International Symposium on Computer Aided Control Systems Design, 2004, pp. 284-289.

[16] R. H. Tütüncü, K. C. Toh, and M. J. Todd, "Solving semidefinitequadratic-linear programs using SDPT3," Mathematical Programming Series B, vol. 95, no. 2, pp. 189-217, 2003.

[17] D. Hug, "Random polytopes," in Stochastic Geometry, Spatial Statistics and Random Fields: Asymptotic Methods (Lecture Notes in Mathematics), E. Spodarev, Ed. Berlin Heidelberg, Germany: SpringerVerlag, 2013, pp. 205-238 